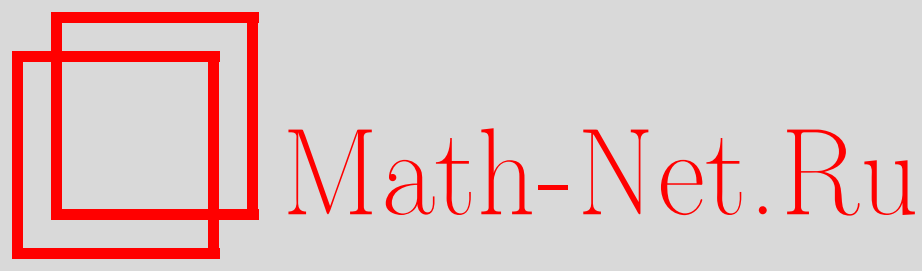

С. С. Герштейн, А. А. Логунов, М. А. Мествиришвили, Гравитационные волны в релятивистской теории гравитации, ТМФ, 2009, том 160, номер 2, 270-275

DOI: https://doi.org/10.4213/tmf6397

Использование Общероссийского математического портала Math-Net.Ru подразумевает, что вы прочитали и согласны с пользовательским соглашением http://www.mathnet.ru/rus/agreement

Параметры загрузки:

IP: 44.207 .124 .84

26 апреля 2023 г., 15:25:44

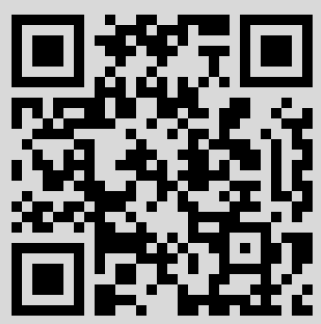




\section{ГРАВИТАЦИОННЫЕ ВОЛНЫ В РЕЛЯТИВИСТСКОЙ ТЕОРИИ ГРАВИТАЦИИ}

Показано, что в релятивистской теории гравитации с гравитоном, имеющим массу покоя, гравитационные волны благодаря условию причинности не имеют нефизических "духовых" состояний.

Ключевые слова: масса покоя гравитона, гравитационные волны.

В работах [1], [2] показано, что в линеаризованной теории гравитации введение массы покоя гравитона в поле со спинами 2 и 0 приводит при объяснении гравитационных эффектов в Солнечной системе к появлению нефизических "духовых" состояний, обязанных спину 0. "Духовые" состояния появляются и в гравитационном излучении, поэтому сложилось мнение, что это доказывает, что масса покоя гравитона точно равна нулю. Однако авторы работ [1], [2] в своих исследованиях не рассматривали гравитационное поле как тензорное физическое поле в пространстве Минковского, создающее своим действием эффективное риманово пространство и, следовательно, свой конус причинности. Именно поэтому не возникло условие причинности, которое имеет место только в релятивистской теории гравитации (РТГ) [3], поскольку в ней имеются два конуса причинности. В общей теории относительности (ОТО) имеется только конус причинности риманова пространства.

В работе [4] при изучении гравитационного излучения было обнаружено, что учет нелинейных членов позволяет устранить “духовые” состояния. Такой подход также разделяли авторы настоящей работы, и он нашел отражение в монографии [3]. Здесь мы покажем, что в РТГ отрицательный поток энергии и, следовательно, "духовые" состояния в гравитационной волне исключаются и без учета нелинейных членов, оказывается, достаточно только соблюдать условие причинности РТГ. Мы, следуя книгам [5]-[7], исходим из возможности существования свободного гравитационного

* Институт физики высоких энергий, г. Протвино, Московская обл., Россия. E-mail: Semen.Gershtein@ihep.ru, Anatoly.Logunov@ihep.ru

${ }^{\dagger}$ Институт теоретических проблем микромира им. Н. Н. Боголюбова; Московский государственный университет им. М. В. Ломоносова, Москва, Россия 
поля - гравитационных волн - как объективной физической реальности подобно электромагнитным волнам в пустоте.

Для простоты и точности анализа рассмотрим в пустоте слабую плоскую гравитационную волну с амплитудой $a^{\mu \nu}(k)$, распространяющуюся вдоль оси $Z$,

$$
\Phi^{\mu \nu}=a^{\mu \nu}(k) \cos k x,
$$

где $k_{\nu}=(\omega, 0,0,-q \omega), q^{2}=1-m^{2} / \omega^{2}, m$ - масса покоя гравитона.

Мы пользуемся системой единиц, в которой $G=\hbar=c=1$. В пустоте основные уравнения РТГ в линейном приближении в инерциальной системе в галилеевых координатах для слабого гравитационного поля имеют вид

$$
\begin{aligned}
\square \Phi^{\mu \nu}+m^{2} \Phi^{\mu \nu} & =0, \\
\partial_{\nu} \Phi^{\mu \nu} & =0 .
\end{aligned}
$$

Волна (1) является решением этих уравнений. Слабое гравитационное поле $\Phi^{\mu \nu}$ создает эффективное риманово пространство с метрическим тензором

$$
g_{\mu \nu}=\gamma_{\mu \nu}-\Phi_{\mu \nu}+\frac{1}{2} \gamma_{\mu \nu} \Phi, \quad \Phi_{\mu \nu} \gamma^{\mu \nu}=\Phi
$$

аналогично выражается и тензор $g^{\mu \nu}$ :

$$
g^{\mu \nu}=\gamma^{\mu \nu}+\Phi^{\mu \nu}-\frac{1}{2} \gamma^{\mu \nu} \Phi
$$

Отсюда следует, что скалярная кривизна $R$ эффективного риманова пространства равна

$$
R=\frac{1}{2} m^{2} \Phi
$$

Но оказывается (это будет видно в дальнейшем), что ее влияние на поток энергии отсутствует. Интервал пространства Минковского в инерциальной системе в галилеевых координатах равен

$$
d \sigma^{2}=\gamma_{\mu \nu} d x^{\mu} d x^{\nu}=d t^{2}-d x^{2}-d y^{2}-d z^{2} .
$$

Поскольку в РТГ гравитационное поле рассматривается как тензорное физическое поле, развивающееся в пространстве Минковского, конус причинности возникшего эффективного риманова пространства не должен выходить за пределы конуса причинности пространства Минковского. Это и есть принцип причинности РТГ. Согласно этому принципу в эффективном римановом пространстве, которое создано физическим полем, времениподобные и изотропные геодезические этого пространства не должны выходить за пределы границы конуса пространства Минковского. Именно этому физическому требованию нам необходимо дать математическую формулировку.

В РТГ в гиперболические динамические уравнения гравитационного поля члены со вторыми производными по координатам пространства-времени входят в форме

$$
g^{\mu \nu} \frac{\partial^{2} \Phi^{\alpha \beta}}{\partial x^{\mu} \partial x^{\nu}} .
$$


Уравнение характеристик гравитационных уравнений определяется только членами с высшими производными (6):

$$
g^{\mu \nu} \frac{\partial S}{\partial x^{\mu}} \frac{\partial S}{\partial x^{\nu}}=0
$$

Это уравнение определяет фронт волны поля, если поле не имеет массы покоя. $X a$ рактеристики определяют конус причинности эфбективного риманова пространства. В уравнении характеристик (7) для каждого члена со второй производной в (6) присутствует соответствующий член. Если в (6) отсутствует какой-то член со второй производной, то в (7) соответствующий член не появится.

Времениподобные геодезические линии в соответствии с (7) описываются уравнениями Гамильтона-Якоби

$$
g^{\mu \nu} \frac{\partial S}{\partial x^{\mu}} \frac{\partial S}{\partial x^{\nu}}=1
$$

Весь набор геодезических линий в соответствии с (6) определяется уравнениями

$$
g^{\mu \nu} \frac{\partial S}{\partial x^{\mu}} \frac{\partial S}{\partial x^{\nu}}=\left\{\begin{array}{l}
0 \\
1 \\
-1
\end{array}\right.
$$

где равенства в первой и второй строках определяют изотропные и времениподобные геодезические линии, тогда как третья строка соотношения описывает пространственноподобные геодезические линии.

Таким образом, на основании (7) и (8) изотропные и времениподобные геодезические линии, соответствующие (6), удовлетворяют неравенству

$$
g^{\mu \nu} \frac{\partial S}{\partial x^{\mu}} \frac{\partial S}{\partial x^{\nu}} \geqslant 0 .
$$

Это неравенство можно записать в форме

$$
g_{\alpha \beta} p^{\alpha} p^{\beta} \geqslant 0,
$$

где контрвариантный вектор $p^{\alpha}$ равен

$$
p^{\alpha}=g^{\alpha \mu} \frac{\partial S}{\partial x^{\mu}} .
$$

Чтобы конус причинности эффективного риманова пространства находился внутри конуса причинности пространства Минковского, необходимо и достаточно выполнить следующее неравенство:

$$
\gamma_{\alpha \beta} p^{\alpha} p^{\beta} \geqslant 0
$$

Неравенства (10) и (12) можно записать в форме, непосредственно связанной с геодезическими движениями (7) и (8), находящимися в точном соответствии с (6):

$$
\begin{aligned}
g^{\mu \nu} p_{\mu} p_{\nu} & \geqslant 0, \\
\gamma_{\alpha \beta} g^{\alpha \mu} g^{\beta \nu} p_{\mu} p_{\nu} & \geqslant 0,
\end{aligned}
$$


где ковариантный вектор $p_{\nu}$ равен

$$
p_{\nu}=\frac{\partial S}{\partial x^{\nu}}
$$

Условия причинности (13), (14) налагают определенные жесткие требования на решения уравнений гравитационного поля. Только решения, удовлетворяющие неравенствам (13), (14), имеют в теории физический смысл. Неравенства (13), (14) непосредственно связаны с гиперболическими уравнениями гравитационного поля, поскольку они установлены на основе структуры вторых производных (6) динамических уравнений. Именно эта математическая формулировка принципа причинности и обеспечивает нахождение риманова конуса причинности внутри конуса причинности пространства Минковского в соответствии с динамической структурой (6).

Ранее в монографии [3] мы осознали не в должной мере факт необходимости установления прямой связи принципа причинности с гиперболической динамической системой уравнений. В случае статической системы уравнения гравитационного поля уже не являются гиперболическими, и поэтому такой прямой связи уже не будет, однако в этом случае условие причинности можно использовать и в виде неравенств (10), (12). Учитывая равенство

$$
\tilde{g}^{\mu \nu}=\tilde{\gamma}^{\mu \nu}+\widetilde{\Phi}^{\mu \nu}
$$

где

$$
\tilde{g}^{\mu \nu}=\sqrt{-g} g^{\mu \nu}, \quad \tilde{\gamma}^{\mu \nu}=\sqrt{-\gamma} \gamma^{\mu \nu}, \quad \widetilde{\Phi}^{\mu \nu}=\sqrt{-\gamma} \Phi^{\mu \nu},
$$

неравенства (13), (14) в инерциальной системе в галилеевых координатах можно переписать в виде

$$
\begin{array}{r}
\left(\gamma^{\mu \nu}+\Phi^{\mu \nu}\right) p_{\mu} p_{\nu} \geqslant 0, \\
\gamma_{\alpha \beta}\left(\gamma^{\alpha \mu}+\Phi^{\alpha \mu}\right)\left(\gamma^{\beta \nu}+\Phi^{\beta \nu}\right) p_{\mu} p_{\nu} \geqslant 0 .
\end{array}
$$

Для волны (1) имеет место следующее уравнение характеристики:

$$
g^{\mu \nu} p_{\mu} p_{\nu}=g^{00}\left(p_{0}\right)^{2}+2 g^{03} p_{0} p_{3}+g^{33}\left(p_{3}\right)^{2}=0 .
$$

Для слабого гравитационного поля и конкретного движения (1) вдоль оси $Z$ неравенство (16) выполняется, если величина $x$, определенная как $x=p_{3} / p_{0}$, ограничена неравенствами

$$
x_{1} \leqslant x \leqslant x_{2}
$$

где

$$
\begin{aligned}
& x_{1}=\Phi^{03}-1-\frac{1}{2}\left(\Phi^{00}+\Phi^{33}\right), \\
& x_{2}=\Phi^{03}+1+\frac{1}{2}\left(\Phi^{00}+\Phi^{33}\right) .
\end{aligned}
$$

Таким образом мы определили совокупность времениподобных векторов, лежащих внутри конуса причинности, который образован характеристиками, полученными из (6), для движения (1), ведущего к метрике (4).

2 Теоретическая и математическая физика, т. 160, № 2, 2009 г. 
Неравенство (17) будет выполняться, если

$$
x_{1}^{\prime} \leqslant x \leqslant x_{2}^{\prime}
$$

где

$$
\begin{aligned}
& x_{1}^{\prime}=2 \Phi^{03}-1-\Phi^{00}-\Phi^{33}, \\
& x_{2}^{\prime}=2 \Phi^{03}+1+\Phi^{00}+\Phi^{33} .
\end{aligned}
$$

Конус причинности эффективного риманова пространства находится внутри конуса причинности пространства Минковского тогда и только тогда, когда выполнены неравенства

$$
x_{1}^{\prime} \leqslant x_{1}, \quad x_{2} \leqslant x_{2}^{\prime},
$$

из которых, учитывая (19), (20), получим

$$
\Phi^{00} \pm 2 \Phi^{03}+\Phi^{33} \geqslant 0 .
$$

Из уравнения (3) находим для волны (1)

$$
\Phi^{00}=q \Phi^{03}, \quad \Phi^{03}=q \Phi^{33} .
$$

Подставляя эти равенства в (22), получим

$$
(q \pm 1)^{2} \Phi^{03} \geqslant 0
$$

Из этого неравенства и решения (1) следует, что

$$
\Phi^{03} \equiv 0,
$$

а поэтому на основании (23) мы можем также записать равенства

$$
\Phi^{00} \equiv 0, \quad \Phi^{33} \equiv 0
$$

Принцип причинности в РТГ выбирает физическое решение уравнений гравитации. Из (25), (26) следует, что продольно-продольные компоненты в решении в форме волны (1) отсутствуют. Именно поэтому в потоке энергии не возникает член вида

$$
R \Phi^{03}=\frac{1}{2} m^{2} \Phi \Phi^{03}
$$

поскольку он тождественно равен нулю. При массе покоя гравитона, равной нулю, равенства (25), (26) обычно получают, используя калибровочные преобразования. Здесь же они следуют из требования принципа причинности. Это и обеспечивает положительность потока энергии в РТГ при наличии массы покоя гравитона.

В РТГ в квадратичном приближении в галилеевых координатах поток гравитационной энергии определяется тензорной величиной [3], [4]

$$
t_{g}^{\epsilon \lambda}=\frac{1}{32 \pi} \gamma^{\epsilon \alpha} \gamma^{\lambda \beta}\left(\partial_{\alpha} \Phi_{\nu}^{\tau} \partial_{\beta} \Phi_{\tau}^{\nu}-\frac{1}{2} \partial_{\alpha} \Phi \cdot \partial_{\beta} \Phi\right) .
$$


Подъем и опускание индексов в поле $\Phi^{\mu \nu}$ осуществляется с помощью метрического тензора $\gamma_{\mu \nu}$. Согласно уравнению (3) для решения (1) имеют место равенства

$$
a^{10}=q a^{13}, \quad a^{20}=q a^{23} .
$$

Учитывая (1), а также равенства (25), (26), (28), на основании (27) после усреднения по времени для волны (1) получим

$$
t_{g}^{03}=\frac{1}{32 \pi} q \omega^{2}\left\{\left(a_{1}^{2}\right)^{2}+\frac{1}{4}\left(a_{1}^{1}-a_{2}^{2}\right)^{2}+\frac{m^{2}}{\omega^{2}}\left[\left(a_{3}^{1}\right)^{2}+\left(a_{3}^{2}\right)^{2}\right]\right\} .
$$

Отсюда следует, что в плотности потока волны (1) присутствуют только поперечнопоперечные компоненты $a_{1}^{1}, a_{2}^{2}, a_{1}^{2}$, а также продольно-поперечные компоненты $a_{3}^{1}$, $a_{3}^{2}, a_{0}^{1}, a_{0}^{2}$, причем последние в потоке энергии (29) умножаются на величину $m^{2} / \omega^{2}$. Продольно-продольные компоненты в волне (1) отсутствуют. Следует отметить, что согласно РТГ в данной задаче можно осуществить непрерывный переход к массе гравитона, равной нулю.

Таким образом, из (29) следует, что в РТГ наличие массы покоя гравитона не приводит к появлению нефизических “духовых" состояний. В РТГ “духовые” состояния не возникают и при объяснении эффектов в Солнечной системе, при этом на расстоянии от источника $r \gg r_{\mathrm{g}}=2 M$ имеет место непрерывный переход к массе покоя гравитона, равной нулю. Масса покоя гравитона в РТГ с необходимостью возникает, если мы начинаем рассматривать гравитационное поле как физическое поле в пространстве Минковского.

Благодарности. Авторы выражают благодарность В. И. Денисову, В. А. Петрову, А. П. Самохину, К. А. Свешникову, Н. Е. Тюрину за ценные обсуждения.

\section{Список литературы}

[1] В. И. Захаров, Писъма в ЖЭТФ, 12:9 (1970), 447-449.

[2] H. van Dam, M. Veltman, Nucl. Phys. B, 22:2 (1970), 397-411.

[3] А. А. Логунов, Релятивистская теория гравитации, Наука, М., 2006.

[4] Ю. М. Лоскутов, ТМФ, 107:2 (1996), 329-343.

[5] Л. Д. Ландау, Е. М. Лифшиц, Теоретическая физика. Т. II. Теория поля, Физматлит, М., 2001.

[6] А. С. Эддингтон, теория относителъности, ГТТИ, Л.-М., 1934.

[7] А. Эйнштейн, "О гравитационных волнах", Работы по теории относительности 1905-1920, Собрание научных трудов в 4-х томах, Т. І, ред. И.Е. Тамм, Я. А. Смородинский, Б. Г. Кузнецов, Наука, М., 1965, 631-646.

Поступила в редакцию 22.12.2008 